\title{
A epidemia de aids no Estado de São Paulo: uma aplicação do modelo espaço-temporal bayesiano completo
}

\author{
The aids epidemic in the State of São Paulo: application \\ of the full Bayesian space-time model
}

\author{
Rogério Ruscitto do Prado ${ }^{1}$ e Euclides Ayres de Castilho ${ }^{1}$
}

\begin{abstract}
RESUMO
O Estado de São Paulo, por compreender aproximadamente 40\% dos casos de aids notificados no Brasil, oferece uma situação propícia para análise espaço-temporal, visando melhor compreensão da disseminação do HIV/aids. Utilizando os casos de aids notificados ao Ministério da Saúde nos anos de 1990 a 2004 para pessoas com idade igual ou superior a 15 anos, tendo como fonte de informação o Sistema de Informação de Agravos e Notificação, Ministério da Saúde, foram estimados os riscos relativos de aids segundo sexo para períodos de 3 anos utilizando modelos bayesianos completos. Os modelos utilizados se mostraram adequados para explicar o processo de disseminação da aids no Estado de São Paulo e evidenciam os processos de feminização e interiorização da doença, além de sugerir que os municípios atualmente mais atingidos se encontram em regiões de pólos de crescimento econômico e possuem população inferior a 50.000 habitantes.
\end{abstract}

Palavras-chaves: Espaço-temporal. Modelos bayesianos. HIV/aids. São Paulo.

\begin{abstract}
The State of São Paulo accounts for approximately $40 \%$ of the AIDS cases notified in Brazil and provides a suitable opportunity for space-time analysis aimed at better understanding of the dissemination of HIV/AIDS. Using the AIDS cases notified to the Ministry of Health between 1990 and 2004 , among individuals aged 15 years or over, and the Ministry of Health's information system for disease notification (Sistema de Informação de Agravos e Notificação, SINAN) as the information source, the relative risks of AIDS over three-year periods were estimated using full Bayesian models, for each gender. The models used were shown to be adequate for explaining the process of AIDS dissemination in the State of São Paulo and demonstrated the growth among females and in small-sized municipalities. They also suggested that the municipalities currently most affected are in regions of economic growth and have populations of less than 50,000 inhabitants.
\end{abstract}

Key-words: Space-time. Bayesian models. HIV/AIDS. São Paulo.

Pioneiro na notificação de casos de aids no Brasil, junto com o Estado do Rio de Janeiro ${ }^{7}$, o Estado de São Paulo foi também pioneiro em políticas de tratamento da doença. No início dos anos 80 ocorreu o primeiro caso de morte por aids no estado ${ }^{19}$ e já em 1986, foi instituída a legislação estadual de controle do sangue e hemoderivados, antes da legislação federal ${ }^{6}$, que torna obrigatória a notificação de casos de aids em todo o Brasil. Desde então, a doença vem apresentando um comportamento diferenciado de acordo com as características econômicas, culturais e sociais de cada região.

O Estado de São Paulo, por compreender aproximadamente $40 \%$ dos casos notificados de aids em todo o Brasil ${ }^{15}$, constitui situação favorável para análise espaço-temporal, visando melhor compreensão da disseminação do vírus.

\footnotetext{
1. Departamento de Medicina Preventiva da Faculdade de Medicina, Universidade de São Paulo, São Paulo, SP

Endereço para correspondência: Dr. Rogério Ruscitto do Prado. Dept ${ }^{0}$ de Medicina Preventiva/FM/USP. Av. Dr. Arnaldo 455, 01246-903 São Paulo, SP. e-mail: ruscittousp@yahoo.com.br

Recebido para publicação em 24/04/2008

Aceito em 28/08/2009
}

Originalmente circunscrita à capital do estado e ao litoral, a aids acometia categorias específicas de indivíduos, em especial homens que fazem sexo com homens e receptores de sangue e hemoderivados. Nos primeiros anos da epidemia o município de São Paulo era responsável pela maior proporção de óbitos no estado (60\% em 1988); todavia, no período de 1988 a 1997 houve declínio na participação da metrópole com crescimento do número de óbitos no interior do estado (63\% em 1997). Ainda assim, os casos de notificação para aids concentraram-se na capital $(44 \%)^{14}$. Os óbitos relacionados à aids começaram a diminuir a partir de 1996 e 1997, período em que a política de distribuição gratuita de medicações anti-retrovirais passou a ser federal, ainda que o Estado de São Paulo já a praticasse anteriormente.

As mulheres, que na década de 1980 não eram consideradas vulneráveis à doença, passaram a ser alvo de atenção das políticas de prevenção e atenção à aids no final dos anos 90, pois neste período a aids foi a primeira causa de morte na população feminina do Estado de São Paulo, na faixa etária dos 20 aos 44 anos. Ações efetivas, públicas e privadas, foram alterando esse perfil dramático, embora a razão estadual de morte por aids entre homens e mulheres tenha chegado a 2,6:1 em 1997. 
No que diz respeito à distribuição segundo classe sócioeconômica, uma análise foi desenvolvida a partir dos marcadores de escolaridade e ocupação dos pacientes com aids. Os resultados indicaram que a transmissão por via sexual foi proporcionalmente mais importante nos indivíduos com escolaridade alta, para ambos os sexos, enquanto que o uso de drogas injetáveis, em ascensão nos anos 90, foi proporcionalmente mais importante entre os estratos socioeconômicos menos favorecidos (menor escolaridade, menor qualificação profissional) ${ }^{14}$. Tal característica indicaria pauperização da epidemia entre os homens; os autores sugerem que entre as mulheres a aids já se caracterizaria como uma doença de pobres desde o seu início, pois a maioria dos casos ocorria entre mulheres de baixa escolaridade (apenas 4\% delas teriam escolaridade superior), com parceiros íntimos usuários de drogas injetáveis.

Especificamente no estudo de doenças transmissíveis, em que as observações são registradas em um conjunto de áreas geográficas no tempo, os modelos espaço-temporais se constituem em ferramentas particularmente importantes, pois permitem estimar associações temporais, espaciais e efeitos de interação; contribuem para a compreensão dos processos de disseminação espacial das doenças ao longo do tempo; e corrigem a imprecisão das taxas estimadas a partir de outros métodos, em especial nos municípios com populações pequenas. 0 uso de médias ponderadas ou o emprego dos estimadores, como os bayesianos, têm permitido corrigir distorções e produzir estimativas suavizadas ${ }^{7}$. Esses modelos possibilitam a identificação de potenciais fatores de risco e permitem uma visualização mais precisa da disseminação espacial das doenças ao longo do tempo ${ }^{18}$.

Modelos próprios para dados observados em áreas geográficas (lattice data) foram desenvolvidos ${ }^{4}$ e aplicados para analisar o padrão de disseminação de sarampo ${ }^{10}$. Anos mais tarde, uma medida generalizada de correlação espaço-temporal foi proposta ${ }^{5}$, evidenciando a importância de diferenciar os efeitos de crescimento in situ - crescimento do número de casos em uma determinada localidade ao longo do tempo - dos efeitos de disseminação espacial - crescimento do número de casos em localidades geográficas próximas. No que diz respeito às aplicações de técnicas de análise espacial ao estudo da disseminação da aids, destacam-se alguns estudos, sendo que o crescimento das taxas da doença são maiores em localidades mais densamente povoada ${ }^{9}$, a migração de militares para áreas de combate aumentaram a disseminação do vírus ${ }^{16}$, a disseminação esteve correlacionada com áreas predominantemente agrícolas ${ }^{17} \mathrm{e}$ com grande migração, cujas estruturas sociais são instáveis e com grande presença de prostituiçã $0^{2021}$. Foram, também utilizados correlogramas espaciais para analisar a disseminação espaçotemporal da aids em quatro regiões dos Estados Unidos, de 1982 a 1990 apresentando diferenças relevantes no crescimento da aids em regiões rurais e urbanas ${ }^{11}$.

Nos países em desenvolvimento, ainda são poucos os trabalhos que exploram as dimensões macrossociais da epidemia de aids. No Brasil, poucos trabalhos enfocaram alguns aspectos da distribuição espacial da doença. Szwarcwald cols ${ }^{18}$ analisaram os casos de aids notificados no município do Rio de Janeiro no período de 1988 a 1996, estudando como se deu a disseminação da aids em quatro subperíodos do estudo, avaliando com isso o crescimento espacial da doença e o crescimento in situ, ou seja, local da doença nas regiões administrativas do município. Foi aplicado o modelo espaço-temporal ${ }^{5}$ e utilizada como variável resposta a taxa de incidência de aids, tanto para a totalidade dos casos notificados, como para os casos da categoria de exposição heterossexual, para os casos masculinos entre homens que fazem sexo com homens e para os casos em mulheres adultas. Foram utilizadas, ainda, diversas ponderações espaciais para verificar a que melhor explicaria o comportamento da disseminação espacial da doença. Os resultados obtidos pelas análises para a totalidade dos casos mostraram que a disseminação da aids nas regiões administrativas do Rio de Janeiro não apresentou componente espacial estatisticamente significativo para a maioria das ponderações espaciais utilizadas e que houve predomínio do crescimento temporal (in situ $)^{18}$.

Este trabalho tem por objetivo avaliar a adequação do modelo espaço-temporal para análise da dinâmica de disseminação da aids segundo áreas geográficas, tomando como exemplo os casos de aids notificados no estado de São Paulo.

\section{MATERIAL E MÉTODOS}

Foram considerados todos os casos de aids notificados ao Ministério da Saúde, por ano de diagnóstico, no Estado de São Paulo, de 1990 a 2004, tendo como fonte de informações o Sistema de Informação de Agravos de Notificação (SINAN) Ministério da Saúde (MS), sendo os dados informados até o ano de 2005, excluindo-se os casos notificados nos anos anteriores a 1990 devido a baixa quantidade de notificações e a partir de 2005 devido ao atraso entre a notificação e o diagnóstico da doença. Os dados notificados de aids foram agregados para cada município segundo município de residência e acrescidas das informações municipais de população estimadas pela Fundação Instituto Brasileiro de Geografia e Estatística (IBGE) disponíveis no site do DATASUS do Ministério da Saúde (www.datasus.gov.br), assim, obteve-se um banco de dados com informações de freqüência de casos por ano de notificação, sexo e faixa etária.

De posse das informações dos casos notificados e das populações de cada município os dados foram somados para períodos de 3 anos para evitar possíveis distorções nas estimativas das medidas de risco de aids, que podem ser afetadas pelo número pequeno de pessoas residentes ou pela mudança de distribuição dos municípios de São Paulo, fato este ocorrido de 1996 (número de municípios no Estado de São Paulo era 625) para 1997 (número de municípios passou para 645).

Os casos notificados de aids no Estado de São Paulo, após agrupados em períodos, foram separados por sexo e consideradas as pessoas com idade igual ou superior a 15 anos.

Os dados foram processados por meio do software SPSS 13.0 (Statistical Package for Social Science) (www.spss.com) e da planilha de dados Microsoft Excel 2003. 
As informações de notificação da aids foram padronizadas pelo método indireto para cada sexo, tendo como referência as notificações ocorridas no Brasil no ano de 2000, último ano censitário, separando-se nas faixas etárias de 15 a 19 anos, 20 a 29 anos, 30 a 39 anos, 40 a 49 anos, 50 a 59 anos, 60 a 69 anos, 70 a 79 anos e 80 anos ou mais. Por apresentar distribuições estatísticas convenientes para ajuste dos modelos ${ }^{2}$, criou-se a razão de morbidade padronizada (RMP) de cada município em cada período para cada sexo, sendo esta medida o valor de casos notificados observados no município dividido pela somatória do número de casos esperados para cada faixa etária se esta tivesse a mesma taxa de notificação da população do Brasil no ano de 2000 para a mesma faixa etária. Para verificar a relação dos casos de aids com o fator socioeconômico foi utilizado o índice de desenvolvimento humano (IDH) de cada município como variável proxys da estratificação social. É sabido que o IDH é uma composição de três componentes da população, um indicador de renda, um indicador de longevidade e um indicador de educação (www.ipea.gov.br).

As RMPs dos casos de aids para cada município do Estado de São Paulo, nos períodos estudados, foram ajustadas com o modelo espaço-temporal bayesiano hierárquico completo, supondo influência global (exchangeable prior) para cada sexo e vizinhança adjacente entre os municípios, visando observar a dinâmica da doença no Estado no período de estudo. Também, foi estimado o percentual de alteração dos riscos relativos ao longo dos 15 anos de estudo.

0 modelo utilizado para ajuste das RMPs é descrito a seguir, e foi realizado com uso do software WinBugs 14.0.

$$
\ln \left(\frac{O_{i t}}{E_{i t}}\right)=\alpha_{i}+\beta_{i} t+\gamma I D H_{i} \quad i=1,2, \ldots, 645 \text { e } t=-3,-2,-1,0,1,
$$

onde: $O_{i t}=$ número de casos notificados de aids no município $i$ e tempo $t$

$E_{i t}=$ número de casos notificados esperados no município $i$ e tempo $t$

$\alpha_{i}=0$ logaritmo natural do risco relativo de notificação de aids do município $i$ no período $t=0$;

$\beta_{i}=$ ritmo de variação no período do logaritmo do risco relativo de notificação de aids no município $i$;

$\gamma=0$ efeito do IDH no risco relativo de notificação de aids no município $i$;

$\mathrm{IDH}_{i}=$ IDH no município $i$.

Após a realização dos ajustes dos modelos, foram criados os mapas da distribuição espacial das RMPs ajustadas de notificação de aids para cada sexo e período de tempo, além de uma listagem dos 50 municípios que apresentaram maiores taxas de crescimento da aids no Estado de São Paulo para cada sexo. Os mapas foram criados utilizando o software MapInfo 8.0.

O estudo foi aprovado pela Comissão de Ética para Análise de Projetos de Pesquisa - CAPPesq da Diretoria Clínica do Hospital das Clínicas da Faculdade de Medicina da Universidade de São Paulo, sob número 409/05.

\section{RESULTADOS}

Os mapas que apresentam os riscos relativos de aids, tanto para o sexo masculino como para o feminino, ajustados pelos modelos (Figuras 1 e 2) mostram que o risco relativo de aids sofreu crescimento local ao longo dos anos, ou seja, aumento do risco dentro de cada município, mas não apresentou crescimento geográfico espacial, pois, não aparecem nos mapas aglomerados de municípios com riscos relativos de aids similares.

A taxa de crescimento da aids para o sexo masculino foi para a maioria dos municípios de São Paulo entre 100\% e 200\% no período de 1990 a 2004, enquanto para o sexo feminino o aumento na taxa foi, em sua maioria de $200 \%$ a $300 \%$. Nenhum município apresentou redução nas taxas de aids.

Pela análise da Tabela 1, é possível verificar, ainda, que os municípios onde se verifica maior crescimento de casos de aids são eminentemente urbanos e se localizam em áreas de influência de pólos econômicos regionais do interior e litoral, para onde se verificou, nos anos 90, o fluxo migratório no sentido do interior paulista ${ }^{1}$. Para o sexo feminino, os resultados apresentados foram similares aos masculinos. Nos três primeiros anos do estudo, os municípios com maiores riscos relativos da doença estavam restritos ao litoral e grandes centros.

Em sua maioria os riscos relativos de aids aumentaram ao longo dos períodos. Já a taxa de aids do sexo feminino aumentou para todos os municípios do Estado de São Paulo, sendo que os municípios que apresentaram maiores aumentos das taxas são, em sua maioria, do interior paulista, e com população inferior a 50.000 habitantes (Tabela 1 e Figura 3).

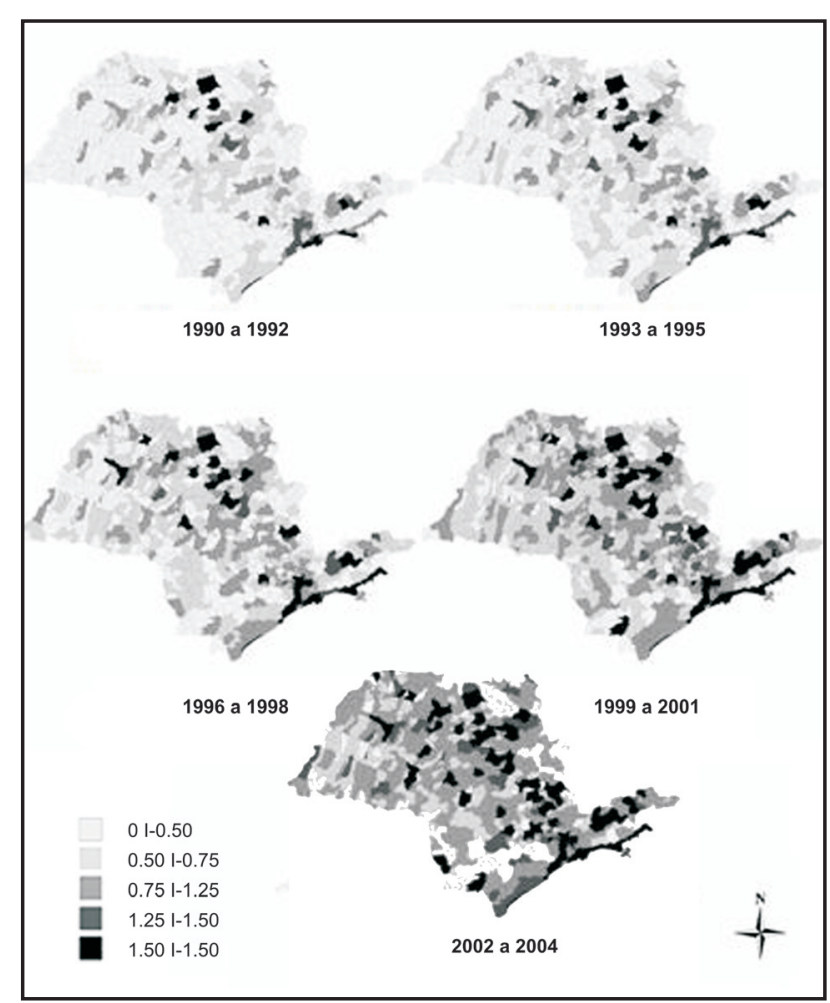

FIGURA 1

Risco relativo de aids para o sexo masculino: Estado de São Paulo, 1990 a 2004. 
TABELA 1

Municípios com maiores taxas de crescimento da aids, segundo sexo. Estado de São Paulo - 1990 a 2004

\begin{tabular}{|c|c|c|c|}
\hline \multicolumn{2}{|l|}{ Masculino } & \multicolumn{2}{|l|}{ Feminino } \\
\hline município & crescimento (\%) & município & escimento (\%) \\
\hline Serra Azul* & 648 & Pitangueiras & 425 \\
\hline Martinópolis & 530 & Itararé & 417 \\
\hline Teodoro Sampaio & 411 & Amparo & 410 \\
\hline Paulo de Faria & 410 & Caraguatatuba & 353 \\
\hline Valinhos & 400 & Santa Bárbara d'Oeste & 349 \\
\hline Itaí & 379 & Sertãozinho & 348 \\
\hline Lucélia & 352 & Itápolis & 347 \\
\hline Pacaembu & 345 & Itatiba & 342 \\
\hline Rancharia & 332 & Itapetininga & 341 \\
\hline Valparaíso & 318 & Lençóis Paulista & 338 \\
\hline Iaras & 308 & São João da Boa Vista & 332 \\
\hline Avanhandava & 299 & Cosmópolis & 331 \\
\hline Pereira Barreto & 294 & Presidente Epitácio & 325 \\
\hline Getulina & 270 & Nova Odessa & 319 \\
\hline Potim & 261 & Teodoro Sampaio & 317 \\
\hline Itupeva & 259 & Salto & 317 \\
\hline Poloni & 250 & Pedreira & 316 \\
\hline Miracatu & 248 & Pompéia & 315 \\
\hline Urupês & 246 & Monte Alto & 307 \\
\hline Barueri & 242 & Tietê & 307 \\
\hline Serra Negra & 242 & Barueri & 305 \\
\hline Conchal & 241 & Pereira Barreto & 300 \\
\hline Lençóis Paulista & 239 & Ibitinga & 300 \\
\hline Itararé & 239 & Cajati & 300 \\
\hline Pirapora do Bom Jesus & 238 & Águas de Lindóia & 299 \\
\hline Piraju & 237 & Louveira & 298 \\
\hline Biritiba-Mirim & 236 & Presidente Venceslau & 297 \\
\hline Meridiano & 234 & Itupeva & 296 \\
\hline Dracena & 234 & São Lourenço da Serra & 296 \\
\hline Piquete & 231 & Artur Nogueira & 293 \\
\hline Junqueirópolis & 230 & Porto Ferreira & 292 \\
\hline Caraguatatuba & 228 & Vargem Grande do Sul & 291 \\
\hline Pilar do Sul & 226 & Ilhabela & 290 \\
\hline Agudos & 225 & Mogi das Cruzes & 290 \\
\hline Areiópolis & 224 & Potim & 289 \\
\hline Bastos & 223 & Álvares Machado & 289 \\
\hline Lavínia & 222 & Iporanga & 288 \\
\hline Descalvado & 221 & Queluz & 287 \\
\hline Quatá & 221 & Pirangi & 287 \\
\hline Pontal & 221 & Capão Bonito & 286 \\
\hline Macatuba & 220 & Vinhedo & 285 \\
\hline Iepê & 220 & Itaí & 284 \\
\hline Itaporanga & 220 & Guareí & 284 \\
\hline Pitangueiras & 218 & Batatais & 284 \\
\hline Guapiaçu & 218 & Araçariguama & 283 \\
\hline Santa Gertrudes & 218 & Populina & 283 \\
\hline Rio das Pedras & 218 & Iguape & 282 \\
\hline Piacatu & 217 & Mirante do Paranapanema & na 282 \\
\hline Amparo & 217 & Morro Agudo & 282 \\
\hline Fartura & 216 & Piratininga & 281 \\
\hline
\end{tabular}

*Os municípios em negrito são os que apresentam menos de 50.000 habitantes.

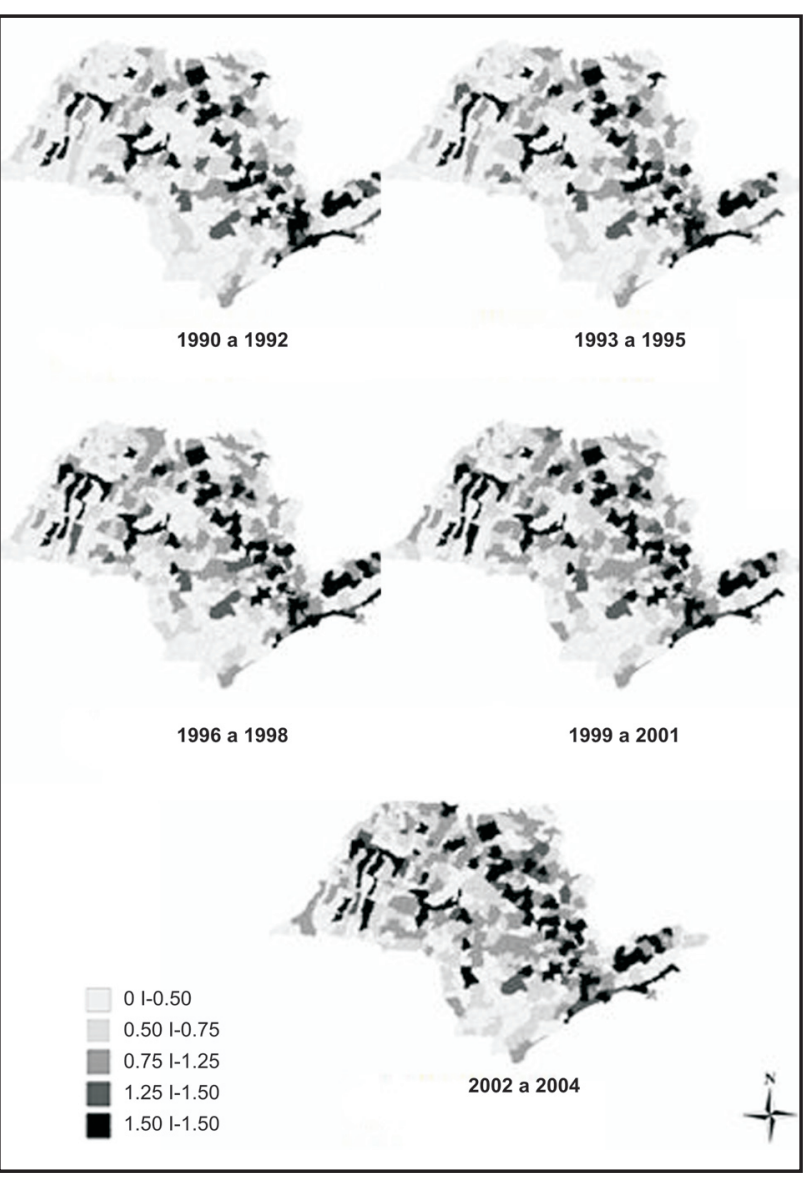

FIGURA 2

Risco relativo de aids para o sexo feminino: Estado de São Paulo, 1990 a 2004.

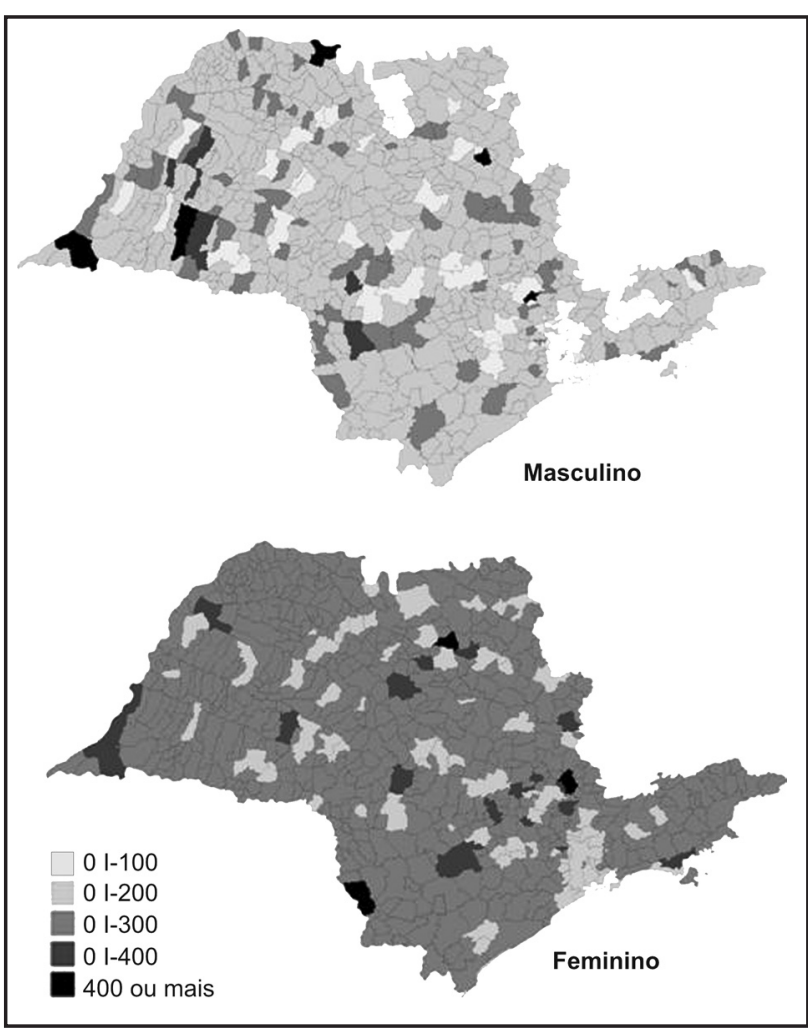

FIGURA 3

Taxas de crescimento de aids segundo sexo: Estado de São Paulo, 1990 a 2004 


\section{DISCUSSÃo}

0 modelo espaço-temporal de análise proposto neste estudo permite explicar a evolução da aids no Estado de São Paulo independentemente de possíveis variabilidades nas taxas de incidência da doença. Com a aplicação dos modelos propostos é possível obter de forma estimada os riscos de aids nos municípios de São Paulo em relação à taxa de incidência nacional - utilizada como referência -, apresentando uma explicação mais clara dos processos de disseminação da doença, nos seus componentes, já relatados em outros estudos, de interiorização e feminização. Quanto ao fenômeno de pauperização, o caso de São Paulo apresenta particularidades, que serão discutidas a seguir.

As estimativas de risco e disseminação dos casos notificados devem fornecer subsídios para a compreensão da evolução da aids no Estado de São Paulo e, com isso, contribuir para formular cenários, e tornar mais efetivas políticas de prevenção e controle da doença.

Os riscos relativos ajustados pelos modelos apresentam maior estabilidade na variabilidade dos casos dentro de cada município e apresentam estimativas mais facilmente interpretáveis.

Tanto para o sexo masculino como para o feminino, a disseminação geográfica espacial da doença não esteve presente, como esperado, e, com isso, o uso do modelo bayesiano completo permutável (exchangeable prior) se mostrou apropriado para explicar a expansão da aids no Estado de São Paulo. Esse resultado corrobora aqueles relativos aos casos de aids no Município do Rio de Janeiro segundo regiões administrativas, em que o crescimento geográfico espacial não foi significativo ${ }^{18}$.

A literatura que abrange o primeiro período da série histórica em estudo já dava conta do processo de interiorização da aids no Estado de São Paulo. Essa tendência foi apresentada nos anos 1987-1996, quando a difusão da doença, visualizada em correlogramas espaciais, indicava a origem da mesma no litoral paulista e na capital do Estado, em direção ao interior, através de rotas de comunicação e transporte, para, finalmente, mostrar uma expansão omnidirecional e generalizada para todo o estado ${ }^{8}$. A teoria da interiorização foi reafirmada em outro estudo ${ }^{19}$, indicando que, em 1997, dos 5.532 óbitos por aids registrados no Estado, 2.044 ocorreram entre residentes da capital, e 3.488 entre residentes do interior. Da mesma forma, foi identificada a difusão da epidemia entre municípios com menos de 50.000 habitantes $^{3}, 0$ que corrobora os resultados obtidos neste estudo, segundo o qual dos cinqüenta municípios em que a aids teve um crescimento mais expressivo entre os homens, quarenta são pequenos, e, entre as mulheres, trinta em cinqüenta municípios de maior crescimento da aids são pequenos, na série histórica estudada.

Os riscos relativos de aids para o Estado aumentaram em quase todos os municípios ao longo do período de estudo (crescimento in situ), independente do sexo, e os municípios do interior paulista passaram a ter riscos relativos de aids bastante elevados nos últimos anos do período, o que mostra que o processo de interiorização da aids, descrito por Brito e cols $s^{3}$ entre outros estudos, está presente nas estimativas de riscos relativos obtidas pelos modelos.
São apresentados neste trabalho os municípios que tiveram maior crescimento de aids nos anos estudados - a maior parte deles com menos de 50 mil habitantes -, localizados em RGs de grandes pólos de desenvolvimento econômico do interior paulista, como Ribeirão Preto, Presidente Prudente, Bauru, Araçatuba, e particularmente nos eixos das rodovias Anhanguera e Washington Luís, vias de comunicação com outros Estados da região CentroOeste e o Estado de Minas Gerais, na região Sudeste.

As estimativas das taxas de crescimento da aids nos municípios do Estado de São Paulo, em sua maioria, foram da ordem de 100\% a $200 \%$ no sexo masculino e de $200 \%$ a $300 \%$ no sexo feminino para o período de 1990 a 2004. Esse resultado vai ao encontro do processo de feminização da aids descrito, por exemplo, por Brito e cols ${ }^{3}$ e mostra que a disseminação da doença entre as mulheres vem ocorrendo de forma superior à ocorrida entre os homens.

0 processo de pauperização da aids, que é a disseminação maior da doença em pessoas provenientes de comunidades mais pobres, desprovidas de autonomia social, política, econômica e cultural, cuja aferição é muitas vezes realizada pela escolaridade das pessoas diagnosticadas ${ }^{312}$ ou pelo índice de desenvolvimento humano, foram relativizados nos modelos ajustados, os quais mostraram que municípios com IDH mais alto apresentam riscos relativos de aids mais altos, e os municípios que apresentam o maior crescimento para aids apresentam IDH elevado [0,7 a 0,8].

No que tange o conceito de feminização da aids, ela é fartamente registrada na literatura sobre o tema, exigindo atenção não apenas naquilo que diz respeito às mulheres propriamente, mas a seus possíveis filhos. Rodrigues Jr e Castilho $\mathrm{EA}^{13}$, entre outros, associam a feminização da aids à vulnerabilidade feminina em relação, entre outras condições, à menor possibilidade de acesso aos serviços de saúde e menor capacidade de negociar sexo seguro ${ }^{12}$. No entanto, cabe considerar no mosaico de epidemias regionais - o que poderá ser aprofundado em estudos futuros - a crescente inserção das mulheres no mercado de trabalho, nos últimos anos, e o já mencionado crescimento do ensino superior, pelo menos no Estado de São Paulo, o que convida a pensar em novas hipóteses para o crescimento da aids entre mulheres.

A questão da feminização é relevante para reiterar a contribuição dos modelos espaço-temporais para a compreensão da evolução da aids. No caso do Estado de São Paulo, as evidências que os mapas apontam, associadas a uma reflexão dos processos mais globais para o território paulista na série histórica em estudo, indicam alternativas mais abrangentes de explicação para a evolução da doença, oferecendo assim subsídios para políticas de prevenção, monitoramento e controle da aids.

Limitações do estudo. Os modelos ajustados não corrigem problemas de informação, como subnotificações, duplicidade ou atraso de notificação, já relatados na literatura ${ }^{12}$, instituindo deste modo uma história da doença na região de interesse.

As relações entre o risco de aids e as hipóteses levantadas sobre a inserção da mulher no mercado de trabalho, a expansão do ensino superior e o crescimento da industria em determinadas 
regiões não podem ser testadas com o modelo implementado por falta dessas informações no momento da execução do estudo, porém, de posse dessas informações o modelo proposto é passível de ajustes que possam incorporá-las.

\section{REFERÊNCIAS}

1. Baeninger R. São Paulo e suas migrações no século 20. São Paulo em Perspectiva 19: 84-96, 2005.

2. Bernardinelli L, Montomoli C. Empirical Bayes Versus Fully Bayesian Analysis of Geographical Variation in Disease Risk. Statistics in Medicine 11: 983-1007, 1992.

3. Brito AM, Castilho EA, Szwarcwald CL. AIDS e infecção pelo HIV no Brasil: uma epidemia multifacetada. Revista da Sociedade Brasileira de Medicina Tropical 34: 207-217, 2001

4. Cliff AD, Ord JK. Spatial Autocorrelation. Pion, London 1973.

5. Cliff AD, Ord JK. Spatial Processes. Models \& Applications. Pion, London 1981.

6. Diário Oficial do Estado de São Paulo. Lei N ${ }^{0}$ 5.190, de 20 de junho de 1986. Dispõe sobre a realização de testes para detecção de anticorpos do vírus da Síndrome da Deficiência Imunológica Adquirida (Aids). Publicação de 21/06/86

7. Dias PRTP, Nobre FF. Avaliando os padrões de difusão espacial. dos casos de aids no Estado de São Paulo. Memórias do II Congresso Latinoamericano de Ingenieria Biomédica, Habana. Sociedad Cubana de Bioingenieria, artículo 00324, La Habana, Cuba, 2001

8. Dias PRTP, Nobre FF. Análise dos Padrões de difusão espacial dos casos de AIDS por Estados brasileiros. Cadernos de Saúde Pública 17:1173-1187, 2001a.

9. Golub A, Gorr WL, Gould PR. Spatial diffusion of the HIV/AIDS epidemic: modeling implications and case study of AIDS incidence in Ohio. Geographical Analysis $25: 85-100,1993$
10. Hagget P. Hybridizing Alternative Models of na Epidemic Diffusion Process, Economic Geography 52:136-146, 1976.

11. Lam N S-N, Fan M, Liu K-B. Spatial-Temporal Spread of the AIDS Epidemic, $1982-$ 1990: A Correlogram Analysis of Four Region of the United States, Geographical Analysis 28:93-107, 1996.

12. Parker R, Camargo Jr KR. Pobreza e HIV/AIDS: aspectos antropológicos e sociológicos. Cadernos de Saúde Pública 16 (supl I): 89-102, 2000.

13. Rodrigues-Júnior AL, Castilho EA. A epidemia de AIDS no Brasil, 1991-2000 descrição espaço-temporal. Revista Sociedade Brasileira de Medicina Tropical 37:312-317, 2004.

14. Santos NJS, Tavra A, Silva SR, Buchalla CM, Laurenti R. A aids no Estado de São Paulo. As mudanças no perfil da epidemia e perspectivas da vigilância epidemiológica. Revista Brasileira de Epidemiologia 5: 286-310, 2002.

15. Secretaria de Vigilância em Saúde. Programa Nacional de DST/AIDS. Dados de Morbidade de Aids, Ministério da Saúde, Brasília, 2005.

16. Smallman-Raynor MR, Cliff AD. Civil war and the spread of AIDS in Central Africa. Epidemiology and Infection 107:69-80, 1991.

17. Smallman-Raynor MR, Cliff AD. Seasonality in tropical AIDS: a geographica analysis. International Journal of Epidemiology 21:547-556, 1993.

18. Szwarcwald CL, Bastos FI, Barcellos C, Esteves MAP, Castilho E. A. Dinâmica da epidemia de AIDS no município do Rio de Janeiro de 1988-1996: uma aplicação de análise estatística espaço-temporal. Cadernos de Saúde Pública 17:1123-1140, 2001.

19. Waldvogel B, Morais LCC. Mortalidade por Aids em São Paulo: dezoito anos de história. XI Encontro Nacional de Estudos Populacionais da ABEP 2131-44, 1998. Disponível em: http://64.233.169.104/search?q=cache:_hyqcl25R4IJ:www. abep.nepo.unicamp.br/docs/anais/PDF/1998/a213.pdf+Mortalidade+por +Aids+em + S\%C3\%A3o+Paulo: +dezoito +anos + de +hist\%C3\%B3ria\&hl=p $\mathrm{t}-\mathrm{BR} \& \mathrm{ct}=\mathrm{clnk} \& \mathrm{~cd}=1 \& \mathrm{gl}=\mathrm{br}$.

20. Wallace R. Social disintegration and the spread of AIDS. Social Science and Medicine 33:1155-1162, 1991.

21. Wallace R. Urban desertification, public health and public order: 'planned shrinkage', violent death, substance abuse and AIDS in the Bronx. Social Science and Medicine 31:801-813, 1993. 